\title{
Research Paper: \\ Socioeconomic Determinants of Disability and Mortality Due to Congenital Anomalies: A Secondary Analysis of Existing Data
}

\section{Behzad Karami Matin ${ }^{1}\left(\mathbb{C}\right.$, Moslem Soofi $^{2}$, Ali Kazemi Karyani ${ }^{1},{ }^{*}$ Shahin Soltani ${ }^{1}{ }^{(0)}$, Bita Shokri $^{3}$, Shiva Amani $^{3}$, Zahra Shahbazi $^{3}$}

1. Research Center for Environmental Determinants of Health (RCEDH), Health Institute, Kermanshah University of Medical Sciences, Kermanshah, Iran 2. Social Development \& Health Promotion Research Center, Health Institute, Kermanshah University of Medical Sciences, Kermanshah, Iran. 3. Students Research Committee, Kermanshah University of Medical Sciences, Kermanshah, Iran.

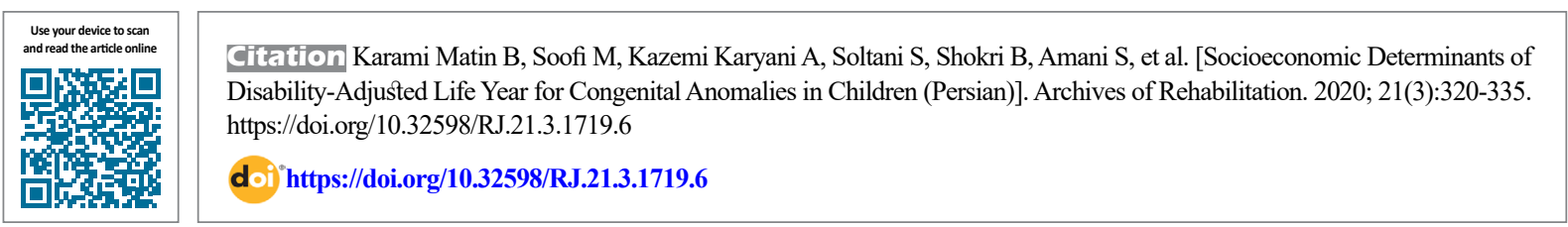

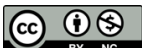

Received: 12 May 2020 Accepted: 04 Aug 2020 Available Online: 01 Oct 2020
Keywords:

Disability, Congenital anomalies, Mortality, Socioeconomic factors poverty

\section{A B STRACT}

Objective Congenital anomalies are functional or structural anomalies that can occur as a single or a group of anomalies. Studies have shown that socioeconomic factors can affect congenital anomalies such that the middle- and low-income countries suffer more from the congenital anomalies. This study aimed to determine the essential socioeconomic determinants of the Disability-Adjusted Life Year (DALY) for congenital anomalies in children aged $<5$ years.

Materials \& Methods This is a cross-sectional study using the latest available data in 2017, which were extracted from the international databases of the World Bank, the Institute for Health Metrics and Evaluation, and the United Nations Educational, Scientific, and Cultural Organization. In the study, we analyzed data from 196 countries divided into 6 geographical regions of African Region (AFRO), European Region (EURO), Pan American Health Organization (PAHO), Eastern Mediterranean Region (EMRO), Western Pacific Region (WPRO), and South-East Asia Region (SEARO). To identify the main determinants of the DALY, Gross Domestic Products (GDP) per capita, poverty rate, government, private and external health expenditures per capita, mean years of schooling, and literacy rate were used. Data analysis was performed in STATA v.15 using the one-way ANOVA and the linear regression analysis.

Results The lowest and highest rates of DALY was reported in Luxembourg (856.29 per 100000 population) and Sudan (21714.7 per 100000 population), respectively. The AFRO and EURO regions had the highest (9392.78 \pm 4250.56$)$, and the lowest (2969.11 \pm 1961.64$)$ mean of DALY. In Iran, the DALY rate was reported 7721.48 per 100000 population, which was higher than those in the EURO and PAHO regions and lower than those in the AFRO and EMRO regions. The results of linear regression analysis showed that mean years of schooling was the strongest predictor of $D A L Y(\beta=-0.44, P=0.001)$ followed by the poverty rate $(\beta=0.36, P=0.002)$. The results of one-way ANOVA indicated that the rate of DALY was significantly different between different social and economic groups, and it was higher in the lower socioeconomic groups. Conclusion Mean years of schooling and poverty rate are the strongest predictors of DALY for congenital anomalies in children under 5 years of age. Low-income countries, especially those in the AFRO and EMRO regions, are the most prone to the disability and premature death caused by congenital anomalies in children under 5 years of age compared to the high-income countries. Therefore, the equitable distribution of screening and health care services and educational infrastructures for deprived and lower socioeconomic countries should be taken into account by national and international health organizations.

\section{* Corresponding Author:}

Shahin Soltani, PhD.

Address: Research Center for Environmental Determinants of Health (RCEDH), Health Institute, Kermanshah University of Medical Sciences, Kermanshah, Iran.

Tel: +98 (918) 3550106

E-Mail: sh-soltani@alumnus.tums.ac.ir 


\section{Extended Abstract}

\section{Introduction}

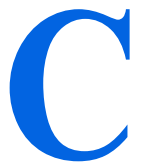

ongenital anomalies include a range of functional or structural anomalies that can occur as a single anomaly or a group of anomalies [1]. The World Health Organization (WHO) estimated that about $6 \%$ of infants worldwide are born with some form of congenital anomaly, and more than 300000 infants aged $<4$ weeks die each year from congenital anomalies [2]. Congenital birth defects are the leading cause of infant mortality, especially in developed countries, whose prevalence has been about $21 \%$ of total infant deaths [3]. In Iran, Vatankhah et al. showed that the overall prevalence of congenital anomalies among infants is about $2.3 \%$, precisely reporting $3 \%$ among boys and $2 \%$ among girls [4]. In a study to identify and record congenital anomalies in Tabriz City, Iran during 2000-2011, Dastgiri et al. reported the overall prevalence of congenital anomalies about 202.9 per 10000 live births [5]. Various factors can affect the occurrence of congenital anomalies. For example, Seddigi et al. showed that consanguineous marriages, contraceptive methods, and male gender could increase the chances of congenital anomalies in infants [6]. Other studies show that family history of genetic disorders, alcohol, and tobacco use during pregnancy, use of unspecified drugs, maternal age, history of abortion, and maternal education level may be associated with an increased risk of congenital anomalies [7-10].

In recent decades, the role of social and economic determinants in the incidence of communicable and non-communicable diseases has been considered by policymakers and researchers in the health system. Social determinants of health can play an essential role in determining the health status of individuals. Variables such as literacy status or education can play an essential role in preventing diseases or disorders and increase people's chances of accessing health care services [11-13]. Studies showed that low-income countries bear a large part of the burden of infectious diseases. With regard to congenital anomalies, studies show that congenital anomalies and their risk factors are mainly seen in people with lower socioeconomic status [14, 15]. For example, Clark et al. in a study in Scotland, showed that the prevalence of orofacial clefts increased with the decrease in socioeconomic status [16].

Table 1. One-way ANOVA results of comparing the mean score of DALY between different social and economic groups

\begin{tabular}{|c|c|c|c|c|c|}
\hline \multirow{2}{*}{\multicolumn{2}{|c|}{ Social and Economic Variables }} & \multirow{2}{*}{ No. } & \multicolumn{3}{|c|}{ Mean $\pm S D^{*}$} \\
\hline & & & DALY & YLD & YLL \\
\hline \multirow{6}{*}{ Region } & AFRO & 45 & $93.78 \pm 42.56$ & $26.31 \pm 59.45$ & $104.64 \pm 48.67$ \\
\hline & EMRO & 23 & $77.88 \pm 46.51$ & $21.89 \pm 29.66$ & $86.24 \pm 53.47$ \\
\hline & EURO & 52 & $29.11 \pm 19.64$ & $23.58 \pm 23.76$ & $31.26 \pm 22.41$ \\
\hline & PAHO & 38 & $49.26 \pm 24.15$ & $170.71 \pm 13.94$ & $54.49 \pm 27.57$ \\
\hline & SEARO & 11 & $58.04 \pm 35.25$ & $245.31 \pm 48.22$ & $63.91 \pm 39.85$ \\
\hline & WPRO & 26 & $52.62 \pm 36.64$ & $211.15 \pm 34.67$ & $57.25 \pm 41.06$ \\
\hline \multirow{4}{*}{ Income } & Low & 30 & $110.74 \pm 44.87$ & $267.51 \pm 68.42$ & $123.88 \pm 51.31$ \\
\hline & Lower than moderate & 46 & $77.55 \pm 37.41$ & $240.24 \pm 42.28$ & $85.87 \pm 42.94$ \\
\hline & Higher than moderate & 57 & $48.86 \pm 22.01$ & $209.36 \pm 38.88$ & $53.43 \pm 26.13$ \\
\hline & High & 61 & $28.17 \pm 15.61$ & $207.17 \pm 34.84$ & $30.26 \pm 18.35$ \\
\hline \multirow{3}{*}{$\begin{array}{l}\text { Mean years of schooling } \\
\text { (Years) }\end{array}$} & $1-5$ & 32 & $11.41 \pm 46.77$ & $261.67 \pm 31.34$ & $123.13 \pm 53.35$ \\
\hline & $6-10$ & 83 & $63.38 \pm 32.98$ & $215.87 \pm 57.75$ & $70.21 \pm 37.02$ \\
\hline & $11-15$ & 74 & $32.43 \pm 20.96$ & $220.62 \pm 37.51$ & $34.74 \pm 23.52$ \\
\hline
\end{tabular}


Table 2. Regression model coefficients for determining the predictors of DALY for congenital anomalies in children aged $<5$ years

\begin{tabular}{ccccc}
\hline Variable & Non-standardized Coefficient & Standardized Coefficient & t & Sig. \\
\hline GDP per capita & 0.01 & 0.07 & 3.45 & 0.592 \\
\hline Poverty & 83.76 & $0.36^{*}$ & 3.22 & 0.002 \\
\hline GHE & -0.47 & -0.11 & -0.84 & 0.4 \\
\hline PVT & 0.53 & 0.05 & 0.46 & 0.64 \\
\hline EXT & -2.28 & -0.02 & -0.32 & 0.74 \\
\hline Mean years of schooling & -628.16 & $-0.44^{*}$ & -3.31 & 0.001 \\
\hline Literacy rate & 11.9 & 0.05 & 0.68 & 0.494 \\
\hline
\end{tabular}

"Significant at $\mathrm{P}<0.05$.

This study investigates the role of socioeconomic variables in determining the degree of disability caused by congenital anomalies. The variable of Disability Adjusted-Life Year (DALY) in children under 5 years of age was used as an outcome variable. Hence, this study aims to compare the DALY for congenital anomalies among different countries and determine its most critical socioeconomic predictors.

\section{Materials and Methods}

This study is a quantitative and cross-sectional study that was performed as a secondary analysis of existing data. The latest data for 2017 were extracted from the databases of the World Bank, the Institute for Health Metrics and Evaluation, and the United Nations Educational, Scientific, and Cultural Organization. Data from 196 countries were analyzed. The countries are divided into 6 geographical regions: African Region (AFRO), European Region (EURO), Pan American Health Organization (PAHO), Eastern Mediterranean Region (EMRO), Western Pacific Region (WPRO), and South-East Asia Region (SEARO), according to the World Health Organization. There were 45 AFRO countries, 23 EMRO countries, 11 SEARO countries, 27 WPRO countries, 55 EURO countries, and 41 PAHO countries. The DALY is expressed per 100000 children less than 5 years of age. The DALY index consists of two components: the Years Lost due to Disability (YLD) and the Years of Life Lost (YLL) due to dying early. Economic determinants included Gross Domestic Product (GDP) per capita (in 2017), poverty, and health expenditures. Health expenditures are divided into three groups: Government Health Expenditure (GHE) per capita, Private health expenditure (PVT) per capita, and External health expenditure (EXT) per capita. The poverty rate is considered the proportion of the population that falls below the poverty line [17]. GHEs are the expenditures incurred by governments and spent from government resources in the health system. PVTs are the expenditures spent by non-governmental resources in the health system, such as expenses paid by patients out of pocket. Finally, EXTs are the expenditures spent from external resources in the health system, such as financial aids from international organizations [18]. The literacy rate (for population over 15 years of age) and mean years of schooling are also examined as two social determinants of health for each of the study countries. Data analysis was performed using one-way ANOVA for comparing the mean score of DALY between different groups and linear regression analysis for determining the predictors of DALY for congenital anomalies in children less than 5 years of age.

\section{Results}

In this study, the available data from 196 countries were examined. The DALY rate due to congenital anomalies in 2017 for children aged $<5$ years in Iran was about 7721.48 per 100000 population. It was higher than the average DALY rate in EURO and PAHO countries and was almost equal to the average DALY rate reported in the EMRO region. The largest amount of this rate was related to the YLD component. The lowest DALY rate was associated with Luxembourg (856.29 per 100000 population), and the highest rate (21714.7 per 100000 population) was related to Sudan. Based on the regions, AFRO Region had the highest mean rates of DALY $(9392.78 \pm 4250.56)$, YLD (266.31 \pm 59.45), and YLL (104.64 \pm 48.67$)$, while the EURO region had the lowest mean rates of DALY $(2969.11 \pm 1961.64)$, YLD $(31.26 \pm 22.41)$, and YLL (170.71 \pm 13.94$)$ compared to other regions (Table 1).

The results of one-way ANOVA showed that the distribution of DALY, YLD, and YLL rates was significantly different between the 6 regions, income groups, and 
schooling groups. The distribution of YLD and YLL varied significantly among income groups and were higher in low-income countries. Moreover, the DALY rate was higher in countries with lower literacy rates, and the difference between them was statistically significant. According to the standard coefficients in the linear regression model, the mean years of schooling $(\beta=-0.44, \mathrm{P}=0.001)$ was the strongest predictor of DALY for congenital anomalies in children aged $<5$ years followed by poverty rate $(\beta=0.36$, $\mathrm{P}=0.002$ ) such that with the increase in the poverty rate, the DALY rate significantly increased (Table 2). Other study variables had no statistically significant relationship with the DALY rate.

\section{Discussion and Conclusion}

Mean years of schooling and poverty were the strongest predictors of DALY for congenital anomalies in children under 5 years of age. This finding shows that the level of education and literacy is an important and significant factor in determining the rate of disability and premature death due to congenital anomalies in these children. The average level of education in the AFRO and EMRO regions is significantly lower than that in the other areas. In these countries, due to factors such as lack of income and educational infrastructure, there are fewer opportunities to study or continue to study, and this factor can be useful in recognizing and preventing congenital anomalies. UNESCO reported that approximately one-fifth of children between the ages of 6 and 11, and one-third of adolescents between the ages of 12 and 14 in sub-Saharan Africa are out of school [19]. This finding can highlight the role of social determinants of health in health status and prevention of congenital anomalies. In previous studies, a lack of health awareness and knowledge has been identified as the most critical barriers to access health care services [20].

In line with the results of previous studies, our results reported that the low level of education was one of the most critical risk factors of congenital anomalies. Taye et al. showed that low maternal education was one of the significant risk factors for congenital anomalies in Ethiopia [7]. Pawluk reported that the prevalence of consanguineous marriage, early marriage, and having more than 4 children were higher in people with low socioeconomic status. These factors increased the risk of congenital anomalies in these groups [21]. Our analysis revealed that African countries are most likely to have congenital anomalies in children under 5 years of age. Consistent with this finding, the Global Burden of Disease Study 2016 showed that countries with poorer social and demographic indicators had higher rates of congenital anomalies [14]. Sitkin et al. also showed that the lack of prenatal diagnostic tools and legal protections for abortions with congenital anomalies in low-income countries could lead to a high incidence of congenital anomalies in these countries [22]. Liu et al. in 2015 reported that in low- and very low-income countries with the highest under-5 mortality rates (e.g. Angola, Central African Republic, Chad, Mali, Nigeria, Sierra Leone, and Somalia). The congenital anomaly was a significant cause of death in children under 5 years of age [23]. In these countries, polio, malaria, and diarrhea are the three leading causes of death under five. In countries with the lowest under-5 mortality rates (such as the EURO countries), congenital anomalies are also the leading cause of death [15]. Olusanya et al. reported that the 4 disorders of epilepsy, mental retardation, and visual and auditory impairments are responsible for 28.9 million (19.9\%) of the healthy years lost due to disability in children and adolescents.

In low-income countries where the government accounts for a smaller share of health costs, people have less access to governmental screening and health care services [25]. Governments, as health care providers, have an essential role in promoting the health of people; lack of funding for health care by governments can endanger the health of all people. Providing prevention and screening services is one of the essential tasks of governments in providing health services which, if left ignored, could endanger the health of individuals and incur irreparable costs to the health systems and economies of countries. This study suggests that equitable distribution of screening services and health care and educational infrastructure for deprived and low socioeconomic groups should be on the agenda of international organizations and national health systems. Providing screening services can effectively reduce health costs, the occurrence of congenital anomalies, and their mortality rates in children under 5 years of age. Since our study used the latest data available in the mentioned databases, some data were not available for some variables and countries during the study period. Therefore, we inevitably used data from previous years. The poverty rate was an economic variable because the complete data were related to this index.

Mean years of schooling and poverty are the strongest predictors of DALY for congenital anomalies in children under 5 years of age. Low-income countries, especially those in the AFRO and EMRO regions, are the most prone to disability and premature death caused by congenital anomalies among children under 5 years of age. 


\section{Ethical Considerations}

Compliance with ethical guidelines

This study was approved by the Ethics Committee of the Kermanshah University of Medical Sciences (Code: IR.KUMS.REC.1399.182).

Funding

This research was supported by the Kermanshah University of Medical Sciences (No. 990365).

Authors' contributions

Conceptualization and supervision: Shahin Soltani and Behzad Karami Matin; Methodology: Ali Kazemi Karyani and Moslem Soofi; Investigation, writing - original draft, and writing - review \& editing: All authors; Data collection: Bita Shokri, Shiva Amani, Zahra Shahbazi; Data analysis: Ali Kazemi Karyani and Moslem Soofi; Funding acquisition and Resources: Behzad Karami Matin and Shahin Soltani.

Conflict of interest

The authors declared no conflict of interest. 


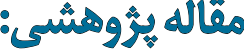

\section{تعيين كنندهالى اجتماعى اقتصادى ناتوانى و مرتى و مير زودرس ناشى از ناهنجارىهاى مادزادى:

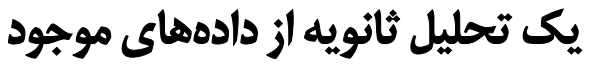

بهزاد كرمى متين' ه، مسلم صوفى"، على كاظمى كريانى'، "شاهين سلطانى' ه، بيتا شكرى"، شيوا امانى" زهرا شهبازى"

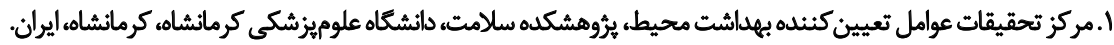

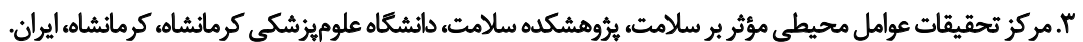

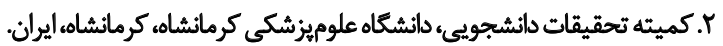

\begin{abstract}
حكיני

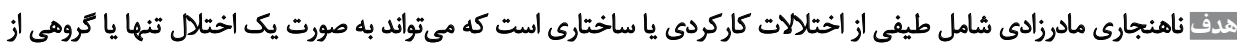

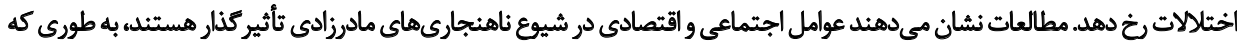

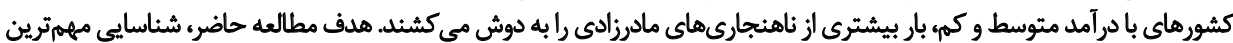

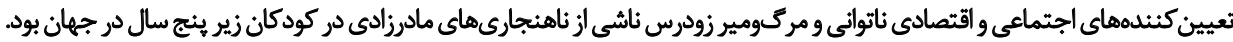

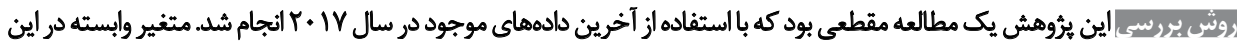

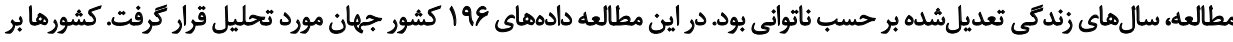

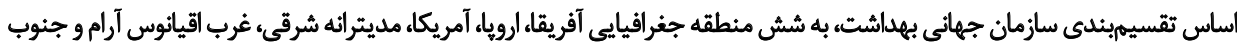

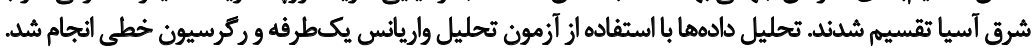

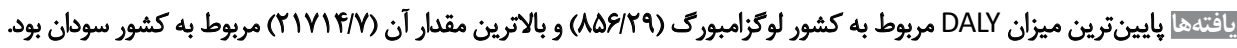

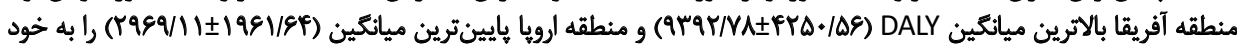

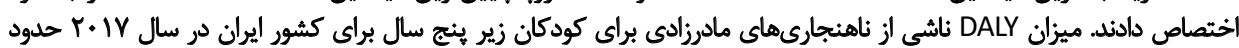

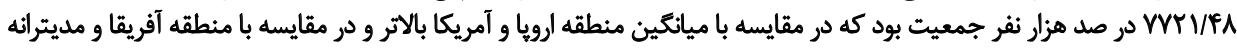

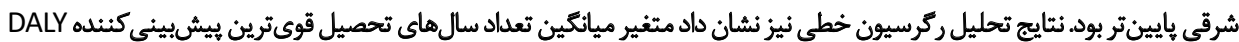

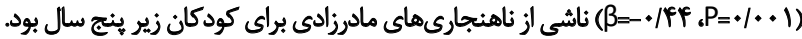

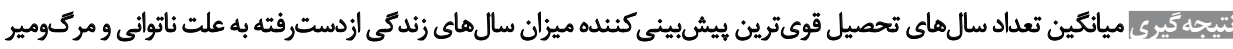

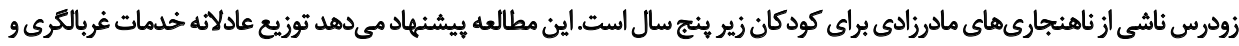

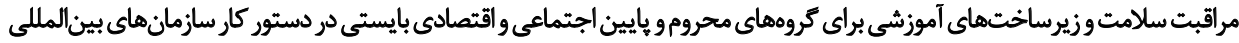
و نظامهاي سلامت ملى قرار كيرد.
\end{abstract}

تاريخدريافت: بrارديبهشت

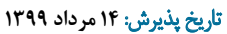

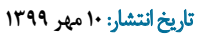

داد. ما در اين مطالعه از اصطلاح ناهنجارى مادرزادى استفاده

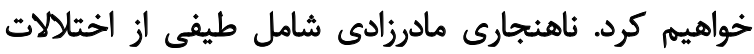

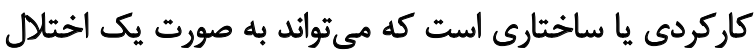

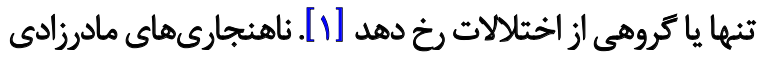

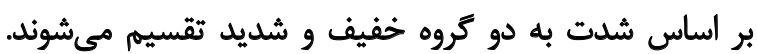

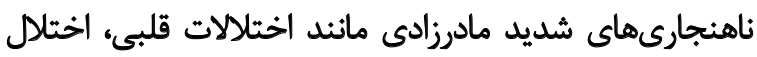

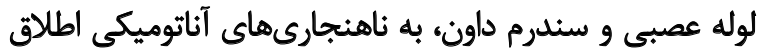

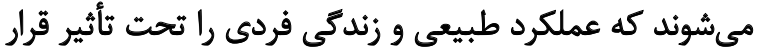

مقلمه ناهنجارى هاى مادرزادى 'كهاز آنهاتحت عناوين بدشكلى مادي

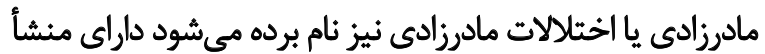

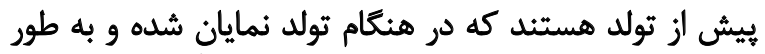
بالقوهاى سلامت نوزاد، رشد يا بقاى او راتحت تولثئ تأثير قرار خواهند

1. Congenital anomalies 
در مصر حاكى از آن بود كه سن بالاى والدين، عوارض دوران

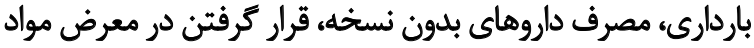

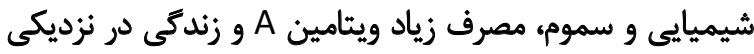

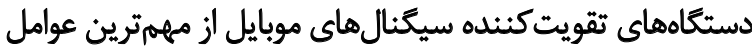

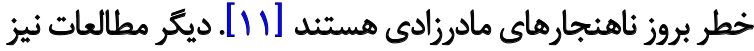

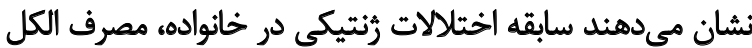

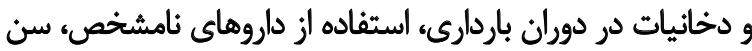

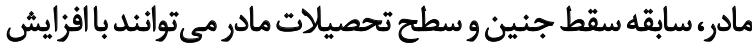

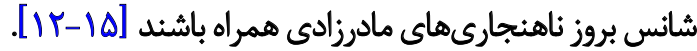

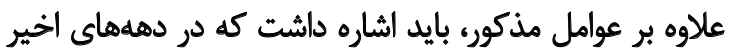

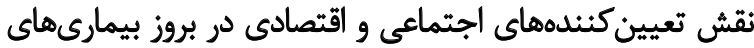

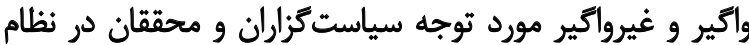

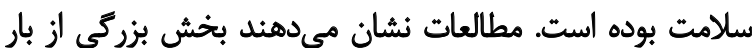

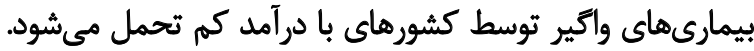

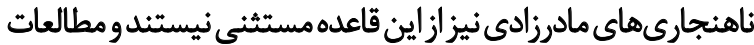

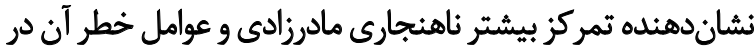

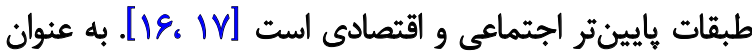

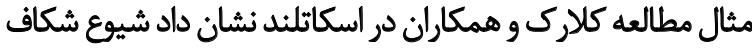

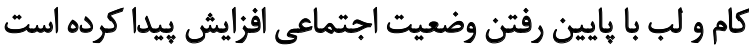

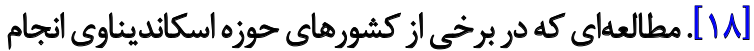

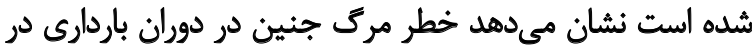

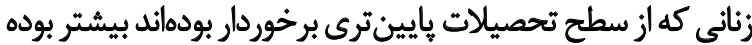

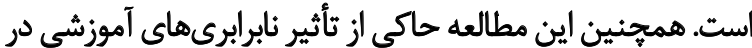

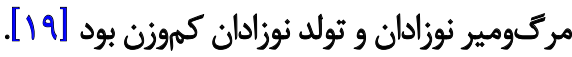

در اين مطالعه ناتوانى ناشى از ناهنجارىهاى مادرزادى با

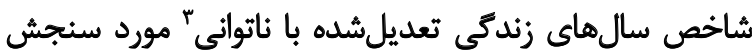

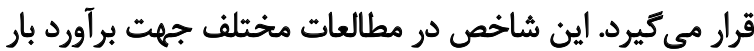

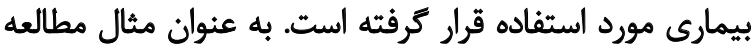

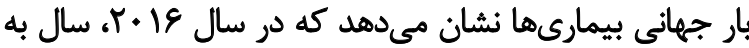

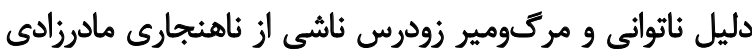

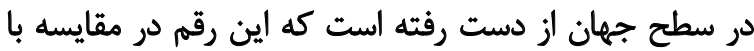

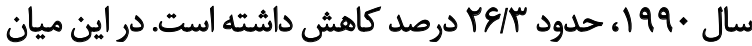

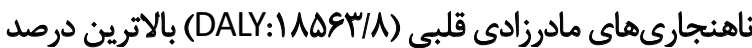

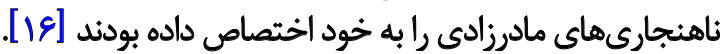
همانطور كه بيان شد شيوع ناهنجارىهاي مادرزادى در

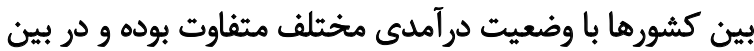

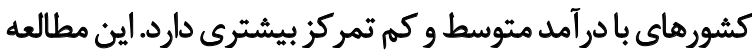

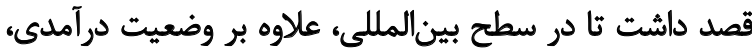
نقش متغيرهاى اجتماعى مانند سواد رانيز در تعيين ميز إن بان ناتوانى

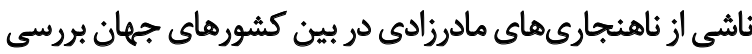

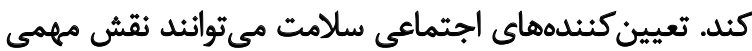

مي دهند، اما ناهنجارىهاى خفيف به تغييرات ساختارى كفته

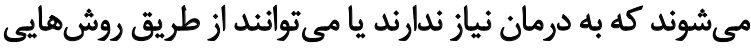

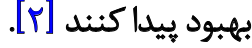
سازمان جهانى بهداشت ب بيان مي كند كه در حدود ع دروصد

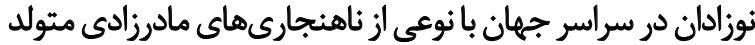

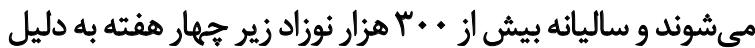

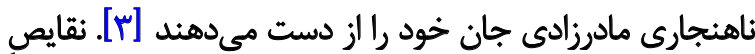

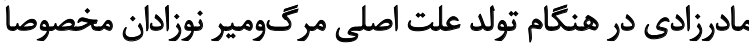

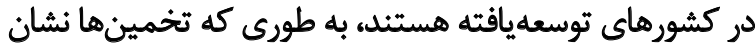

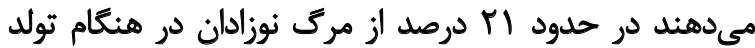

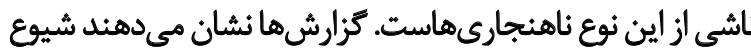

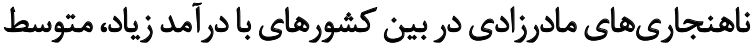

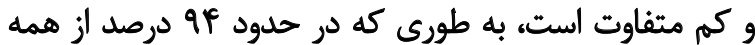

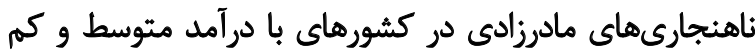

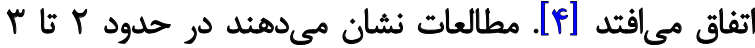

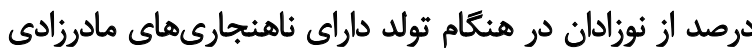

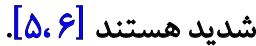

در ايران، مطالعه وطنخواه و همكاران نشان داد شيوع كلى درانى

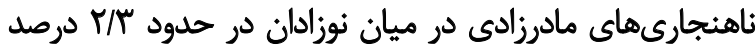

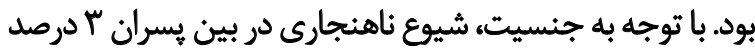

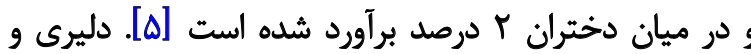

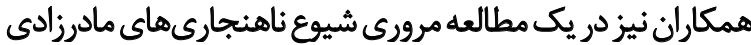

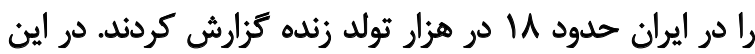

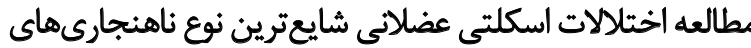

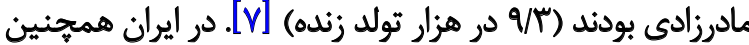

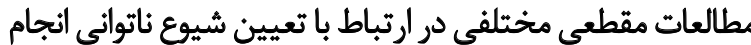

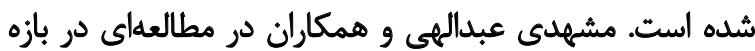

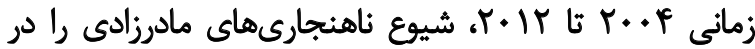
نواحى روستايى تبريز حدود

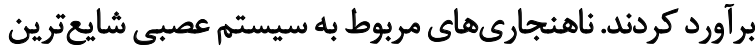

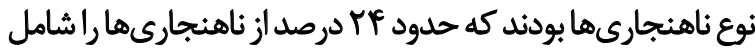

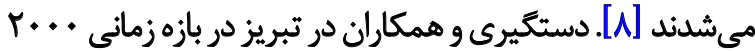

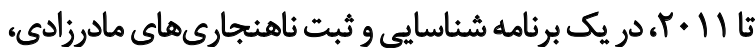

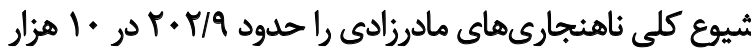

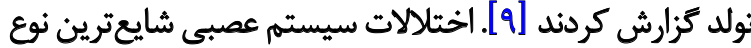

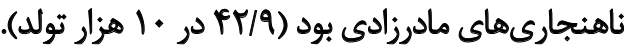
عوامل مختلفى مي توانئد در بروز ناهنجارىهاي مادرزادي

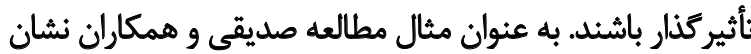

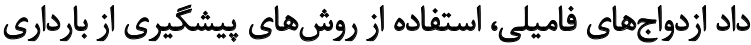

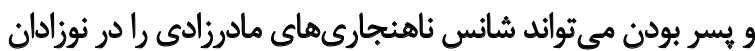

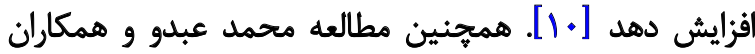



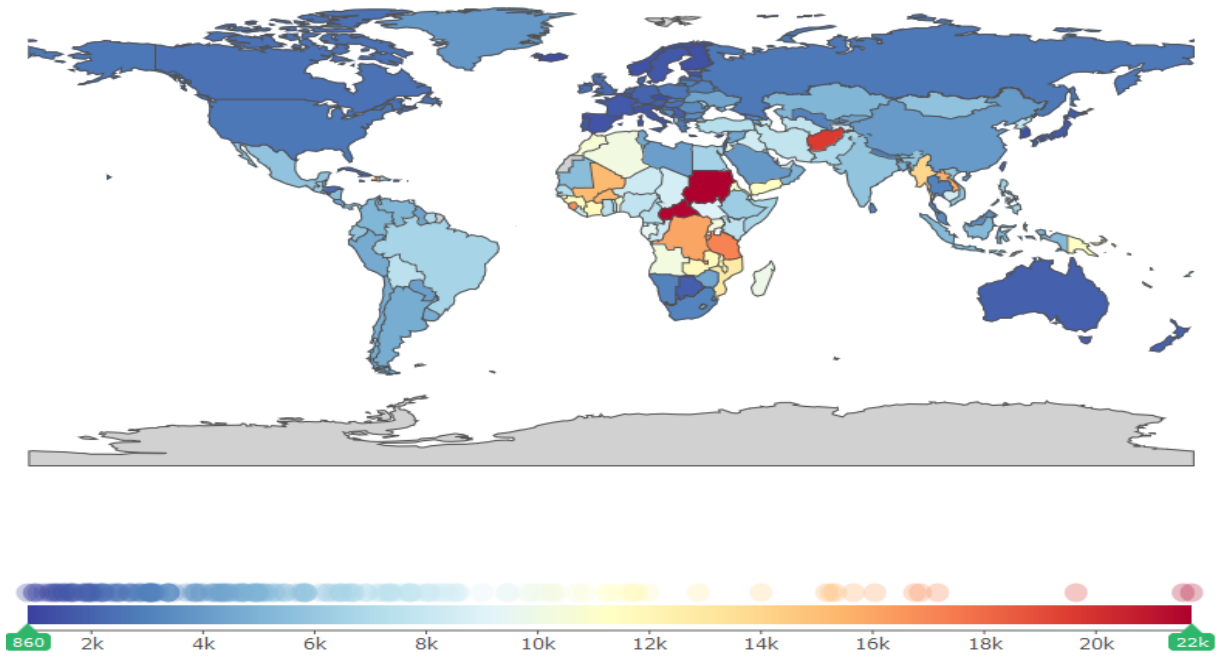

توانبخننى

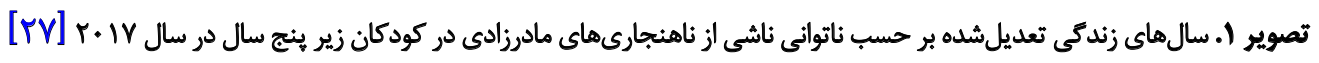

$\mathrm{YLD}=\mid \times \mathrm{DW} \times \mathrm{L}$

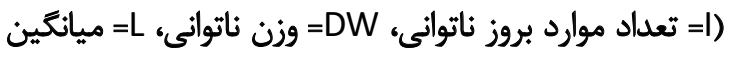

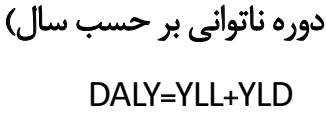

سال بLL) ساى ازدسترفته به دليل مرك زودرس، سال هاى زندگى ازدسترفته به دليل ناتوانى)

طي دهاهاي اخيره استفاده از متغير هاي اقتصادى و اجتماعي

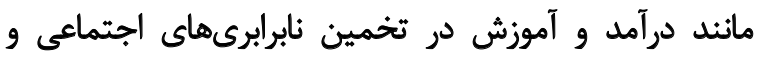
اقتصادى در سلامت اهميت يافته است، به كونهاى كه در در مطالعات مربوط به بار جهانى بيمارىها نقش اقتصاد در وضعيت شيوع

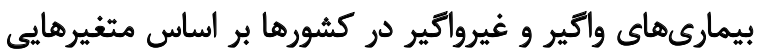

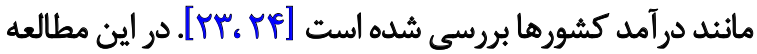

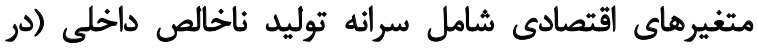

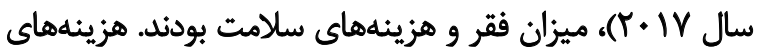

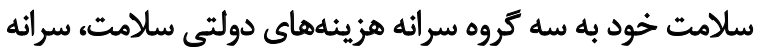

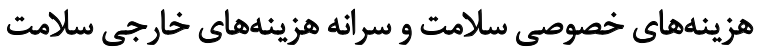

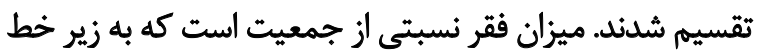

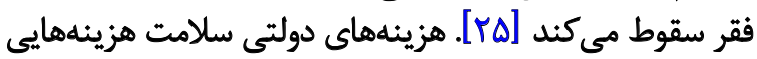
هستند كه توسط دولتها و از مئابع دولتي در نظطام سلامت هزئ هزينه مىشوند. هزينهاى خصوصى سلامت هزينههايى هستند كه از

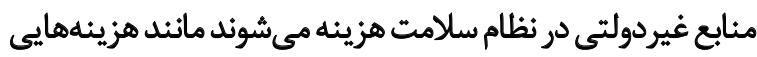

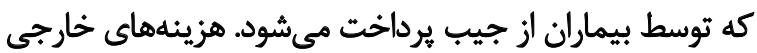

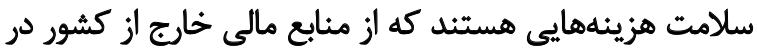

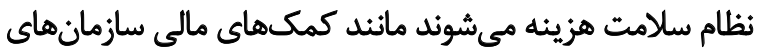

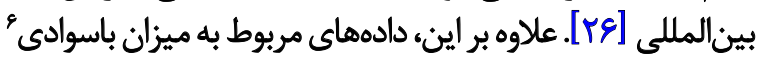

در تعيين وضعيت سلاهت افراد بازى كنئد ميتغيرهايي مانئد

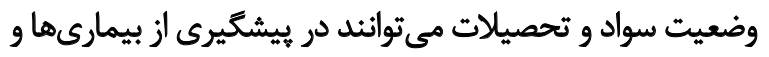

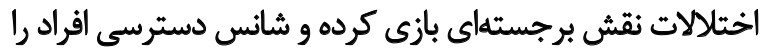

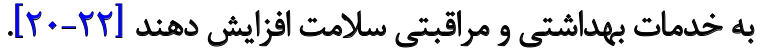

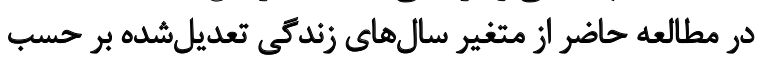

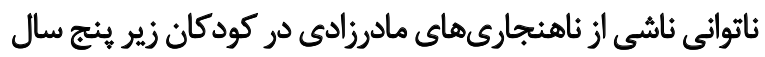

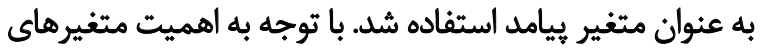

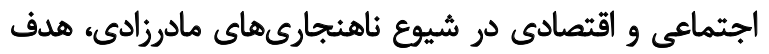

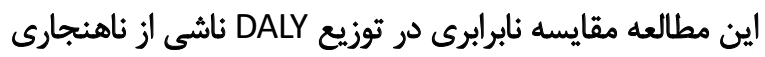

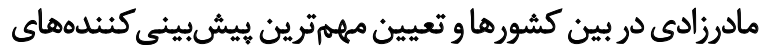
اجتماعي اقتصادي آن بود.

$$
\text { روشّ بروسى }
$$

اين يُوهش يك مطالعه كمى و مقطعى بود كه به روش

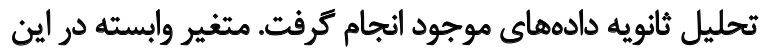

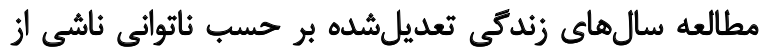

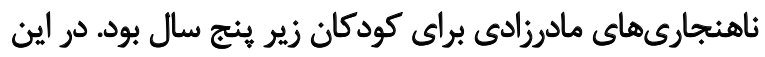

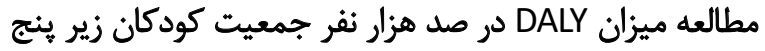

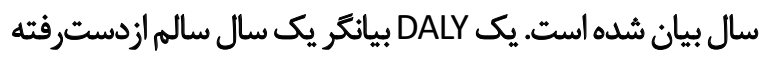

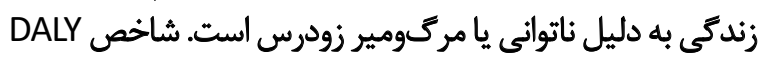

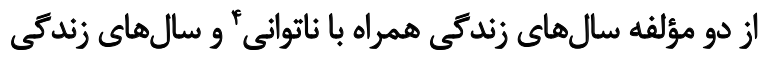

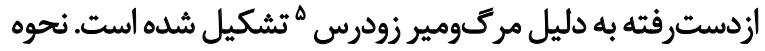

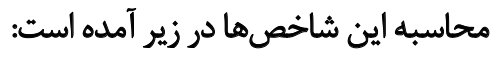

$\mathrm{YLL}=\mathrm{N} \times \mathrm{L}$

(تعداد مركها، =N = اميد به زندكى استاندارد در سن مركى )

4. Years Lost Due to Disability (YLD)

5. Years of Life Lost (YLL) 
جدول ا. تحليل واريانس يكسرفه به منظور مقايسه ميانكين متغير ييامد در بين كروههاي مختلف اجتماعى و اقتصادى

\begin{tabular}{|c|c|c|c|c|c|}
\hline \multicolumn{3}{|c|}{ مياتكين \$|نحرافمعيار" } & \multirow{2}{*}{ فراوانى } & \multirow{2}{*}{\multicolumn{2}{|c|}{ متغير هاى اجتماعى و اقتصادى }} \\
\hline YLL & YLD & DALY & & & \\
\hline $1 . F / R E \pm F N E V$ & $T E \& / T I \pm \Delta q / F \Delta$ & QT/VAETY/AS & is & AFRO & \\
\hline$N E / T P \pm \Delta T / R V$ & 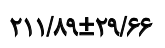 & $W / M \pm F \& / \Delta)$ & r & EMRO & \\
\hline MI/TE士Tr/FI & $r \Psi g / \Delta \Lambda \pm T / V F$ & หม/IIIVAT & $\Delta r$ & EURO & \\
\hline$\Delta P / F q \pm Y V / \Delta V$ & $I V \cdot M \pm I T / q P$ & PQ/RE $\pm T / / \Delta$ & ra & РАНО & منطقه \\
\hline 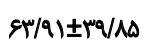 & $T P \Delta / M \pm T N M T$ & $\Delta N \cdot T \pm T \Delta / T \Delta$ & 11 & SEARO & \\
\hline$\Delta V / T \Delta \pm Y V / \circ$ & 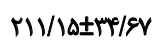 & AY/RTIYE/GE & re & WPRO & \\
\hline$|\pi / M \pm \Delta| / r \mid$ & TEV/OLEENTT & $11 \cdot M \pm \pm r+/ A Y$ & r. & درآمد بايين & \\
\hline $1 \mathrm{~A} / \mathrm{AY} \pm \mathrm{F} T / \mathrm{qP}$ & $T \& \cdot / r \pm \pm T / T \Lambda$ & $W / \Delta \Delta \pm|r|+\mid$ & if & درآمد بايينتر از متوسط & \\
\hline$\Delta T / R T \pm Y R / T$ & $r \cdot q / r E \pm T N M$ & PNAE \pm TY/.1 & ar & درآمد بالاتر از متوسط & $=0$ \\
\hline r./TF士WT' & $r \cdot V / I V \pm M Y / A r$ & YNIVEIQ/PI & \&1 & درآمد بالا & \\
\hline $\mid T T / I T \pm \Delta r / r \Delta \Delta$ & TEV/gYETI/TF & $11 \cdot / F \pm F / W$ & $\pi$ & يك تا ينج سال & \\
\hline$V \cdot / T I \pm T Y / \cdot r$ & $\mathrm{rI \Delta /AY \pm \Delta V/V \Delta}$ & gr/MNATr/U & A & شش تا ده سال & ميانكين سال هاى تحصيل \\
\hline Me/MEtrT/Or & $M Y \cdot|Q Y \pm T V / D|$ & $M T / R+T+/ Q$ & $n$ & يازده ثأ يانزده سال & \\
\hline
\end{tabular}

تحليل دادهها با استفاده از آزمون تحليل واريانس يكسرفه

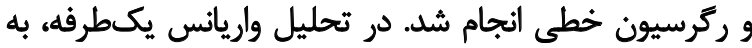

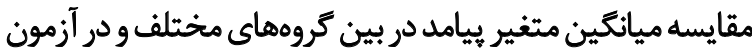

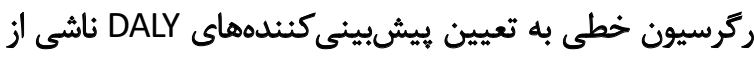

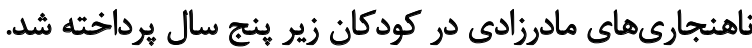

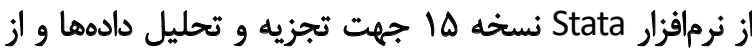

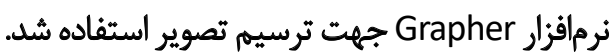

ياقتهلها

در اين مطالعه، نتايج و9 اكشور جهان بر اساس دادههاى موجود

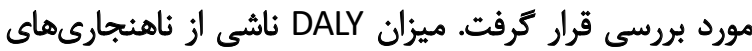

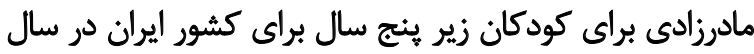

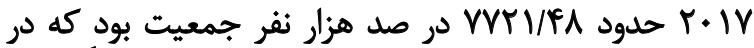

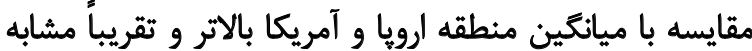

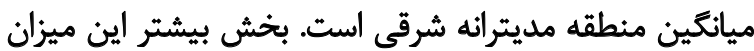

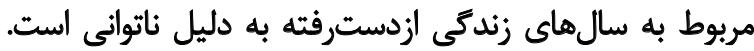

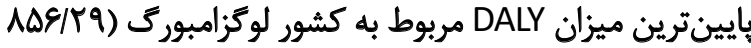

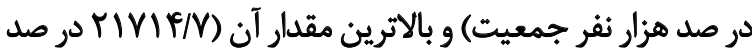

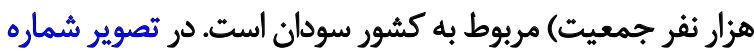

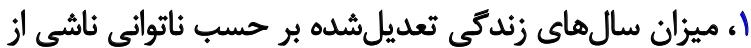

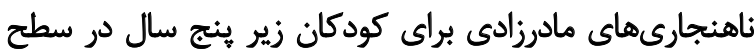

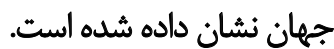

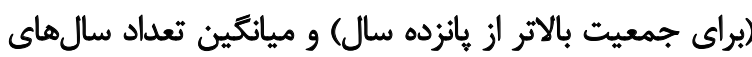

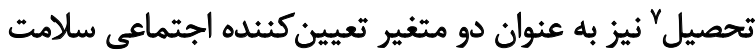

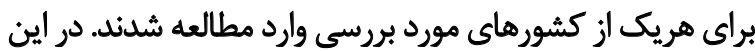

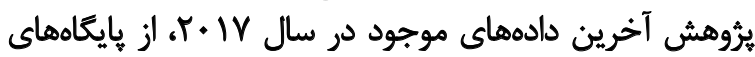

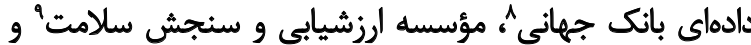

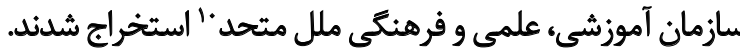

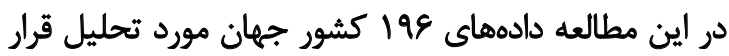

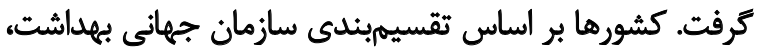

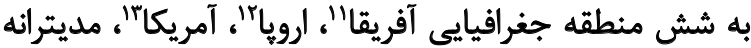

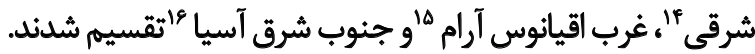

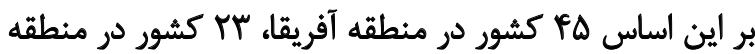

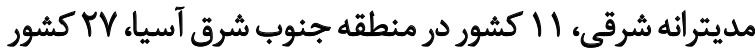

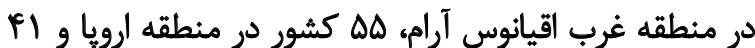
كشور در منطقه آمريكا قرار داشتند.

\section{Mean years of schooling}

8. World Bank

9. Institute for Health Metrics and Evaluation

10. United Nations Educational, Scientific and Cultural Organization

11. African Region (AFRO)

12. European Region (EURO)

13. Pan American Health Organization (PAHO)

14. Eastern Mediterranean Region (EMRO)

15. Western Pacific Region (WPRO)

16. South-East Asia Region (SEARO) 

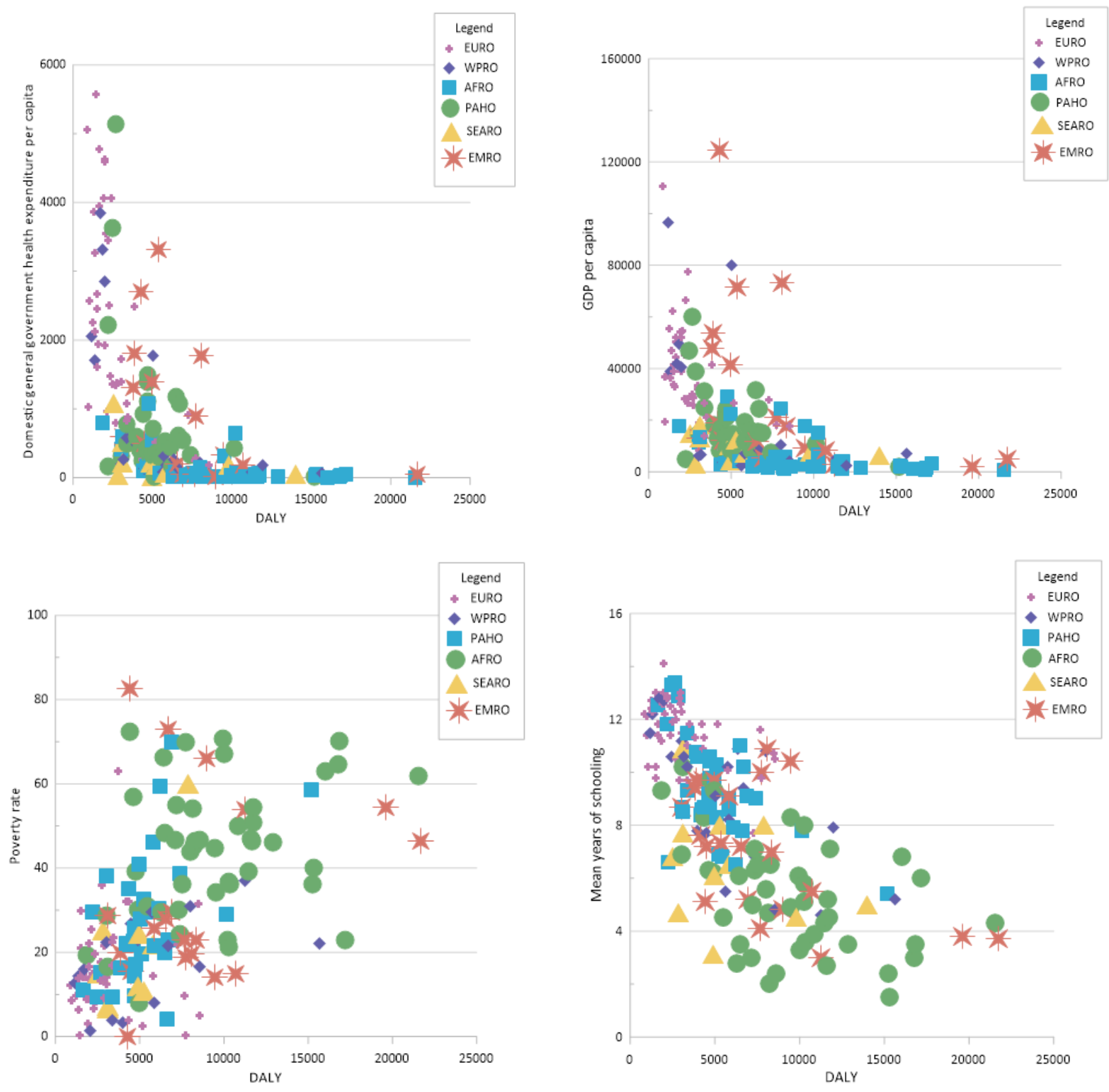

توانبخنتى

تصوير Y. توزيع DALY بر اساس متغيرهاى توضيحى در ميان كشورهاى مورد مطالعه

ناتوائى، در كشورهاى با تحصيلات بإيينتر تمركز بالاترى داشته

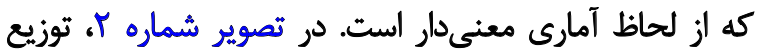

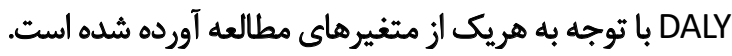
تصوير شماره با، توزيع YLD و وان رادر ميان كشورهاي مورد

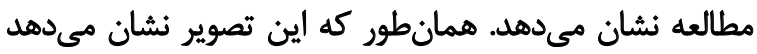

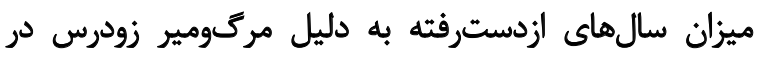

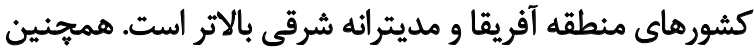

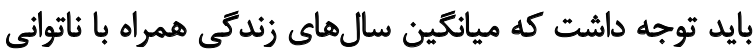
براي كشورهاى منطقه ارويا از همه مناطق به غير ائه ازئ منطقه آفريقا

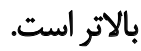

با توجه به ضرايب استاندارد در مدل رگرسيون خطى، متغير
با توجه به جدول شماره ا، منطقه آفريقا بالاترين ميانكين YLL , (r\&\&/TI $\pm \Delta q / F \Delta)$ YLD ( DALY و منطقه ارويا بايينترين ميانكين (1/F/FIFN\&Y)

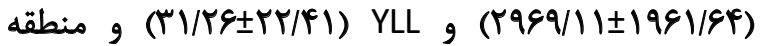
بايينترين ميانكين PAHO

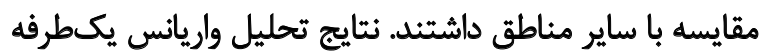

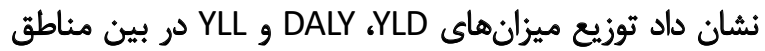

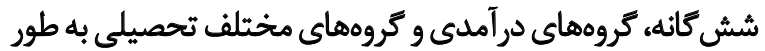

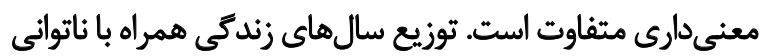

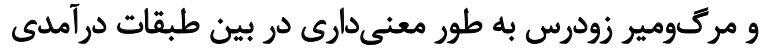

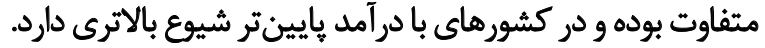

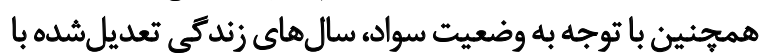


بيشين فقدان آثاهي و دانش سلامت يكى از مهمثترين موانع

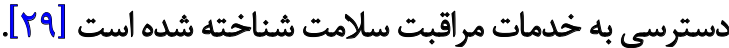

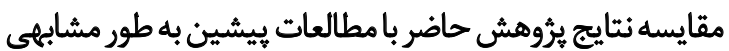

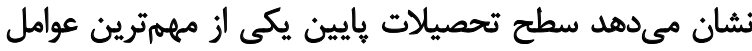

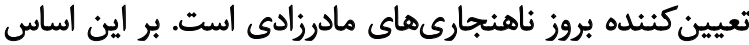

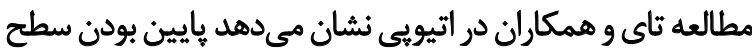

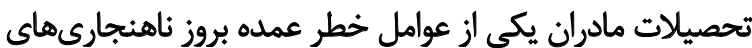

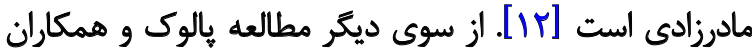

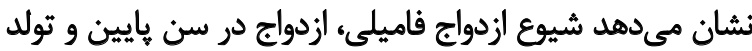

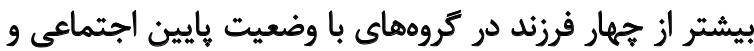

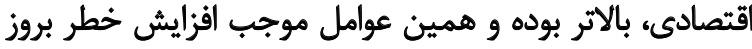

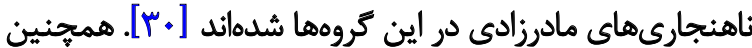

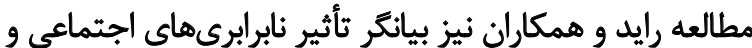

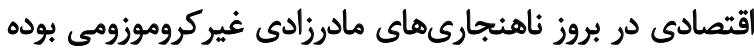

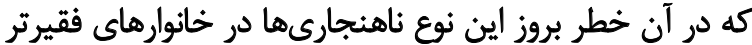

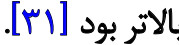

نتايج تحليل واريانس يكطرفه به طور معنى دارى نشان

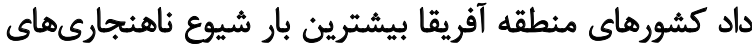

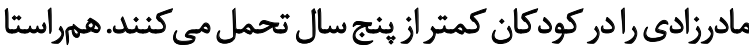

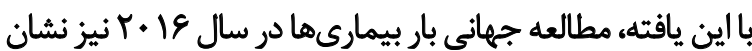

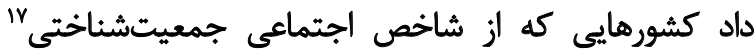

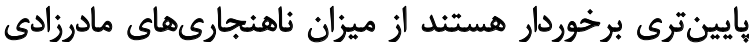

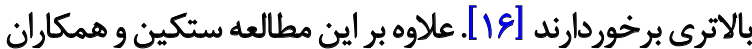

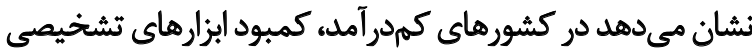
بيش از تولد و نبود حمايتهاى قانونى براى سقط جني جنين دارئي

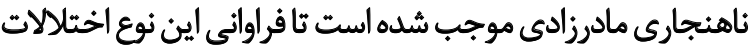

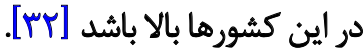

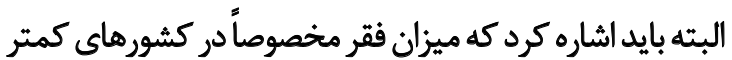

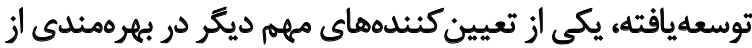

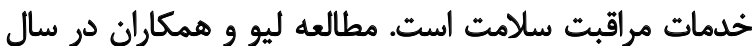

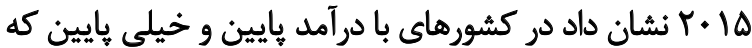

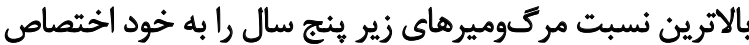

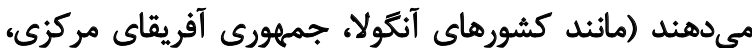

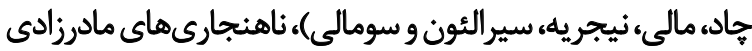

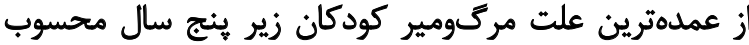

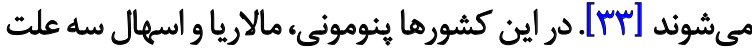

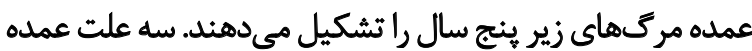

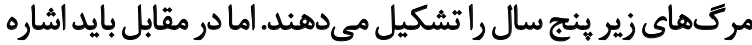

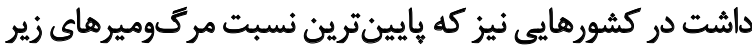

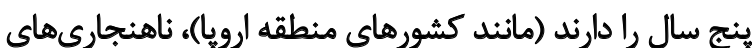

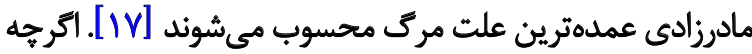

\section{Socio-demographic Index}

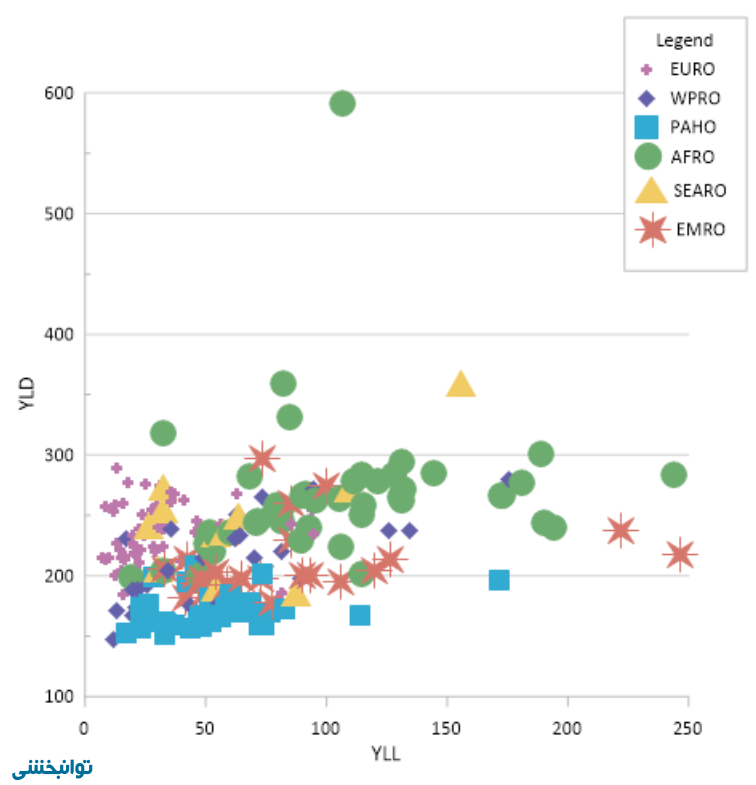

تصوير با. توزيع YLD و دا بين كشور ها بر اساس مناطق مورد مطالعه

ميانكين تعداد سالهاى تحصيل قوىترين بيشبينى

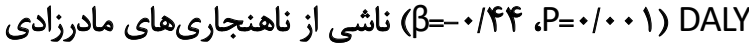

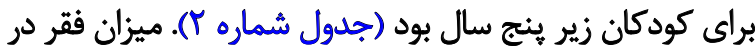

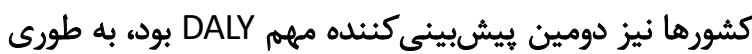

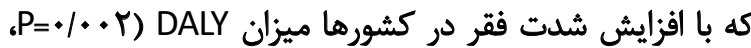

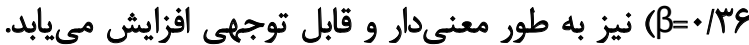

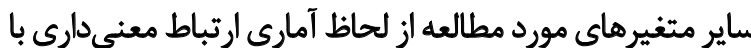

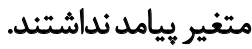
بحث

ميانكين سالهاى تحصيل و ميزان فقر قوىترين

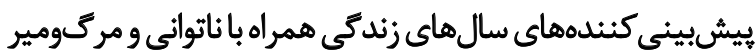

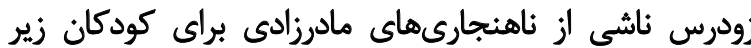

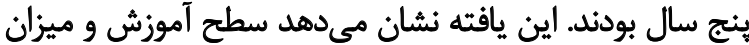

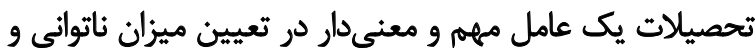

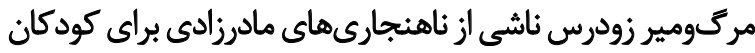

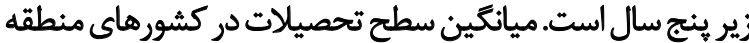

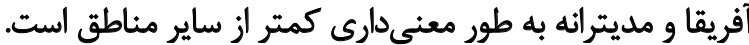

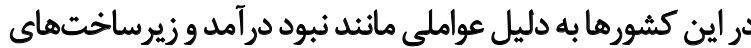

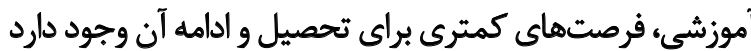

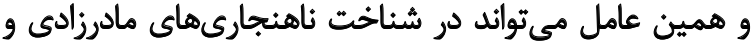

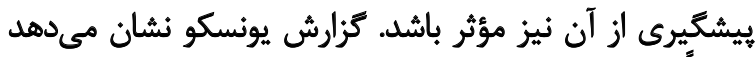

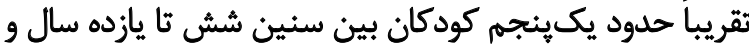

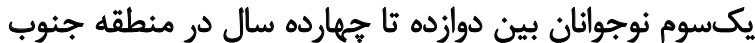

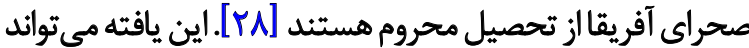

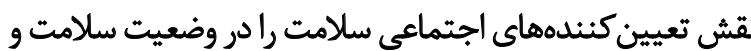

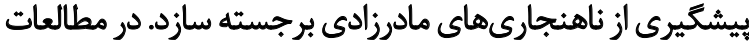


جدول ז. مدل ركرسيون تعيينكنثدهاى اجتماعى و اقتصادى DALY ناشى از ناهنجارى هاى مادرزادى براى كودكان زير بنج سال

\begin{tabular}{|c|c|c|c|c|}
\hline سطح معنى دارى & درجه آزادى (t) & ضرايب استاندارد & ضرايب غيراستاندارد & متثيرها \\
\hline .1094 & $r / * \Delta$ & $\% \vee$ & .1 .1 & سرائه توليد ناخالص داخلى \\
\hline$\%$ & $r / r r$ & $.149^{\circ}$ & $A N / N G$ & ميزان فقر \\
\hline.$/ F$ & $-\cdot / A F$ & $-* / 11$ &.$- / F Y$ & سراثه هزينهاهى دولتى سلامت \\
\hline .194 &.$/ 48$ & .1 .0 &.$/ \Delta r$ & سرائه هزينههاى خصوصى سلامت \\
\hline$\cdot M$ &.$- / \pi$ &.$- / .4$ & $-4 / T \Lambda$ & سرائه هزينههاى خارجي سلامت \\
\hline $.1 . .1$ & - &.$- / \mu \varphi^{\circ}$ & - STNIE & ميانكين تعداد سال هايى تحصيل \\
\hline . /Fqf & .191 & $+1 * 0$ & $11 / 9$ & ميزان باسوادى \\
\hline
\end{tabular}

خدمات دولتى غربالكرى و مراقبت سلامت دارند [حس]]. دولتها

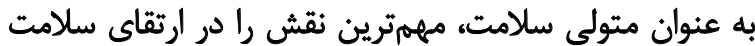

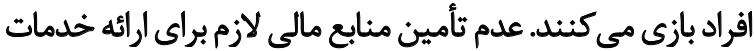

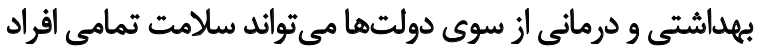

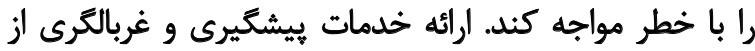

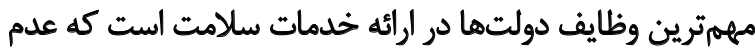

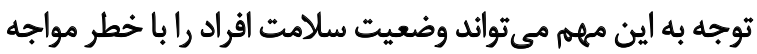

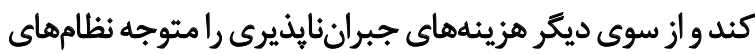
سلامت و اقتصاد كشورها نمايد.

به طور كلى نتايج اين مطالعه نشان مي دهد متغيرهاى ميزان

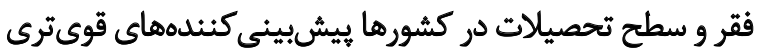

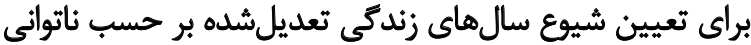

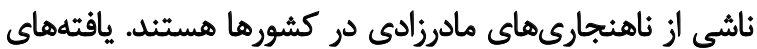

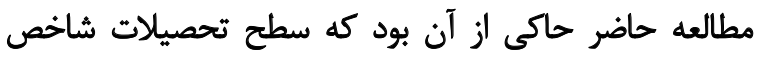

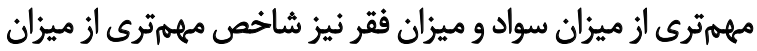

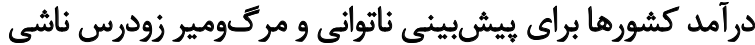

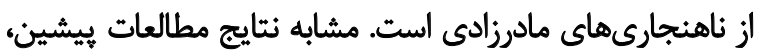

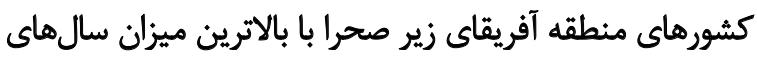

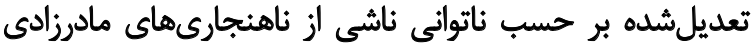

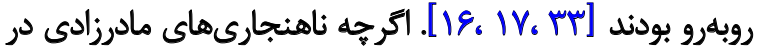

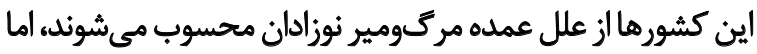

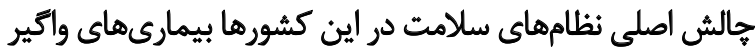

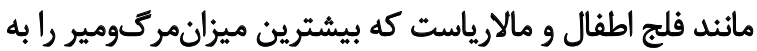

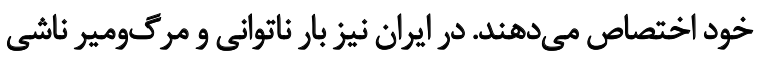

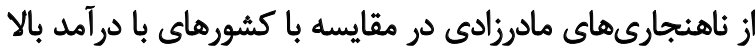

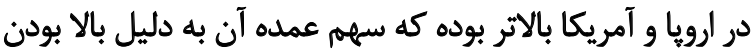

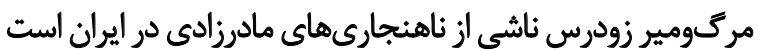

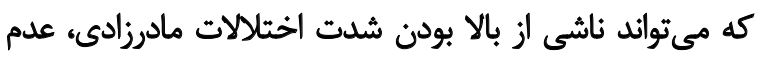

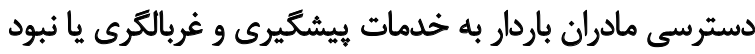

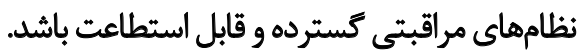

در آفريقاى زير صحرا ميزان ناهنجارىهاى مادرزادى در مقايسه

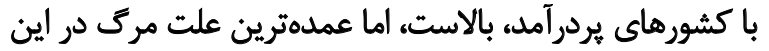

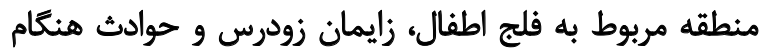

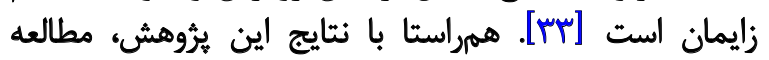

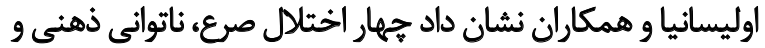
اختلالات بينائى و شنيدارى مسئول TN/

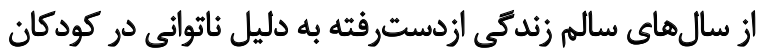

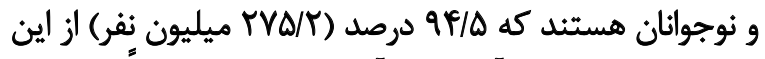

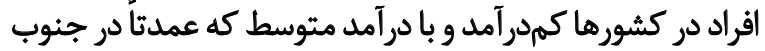

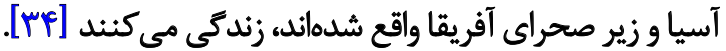

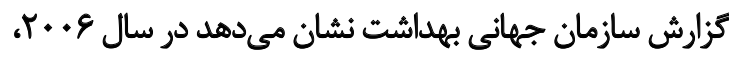
در كشورهايع كمدرآمد (افغانستان، كامبوج، كامرون) كه سيهم

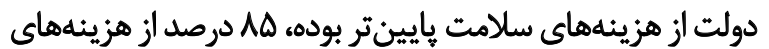

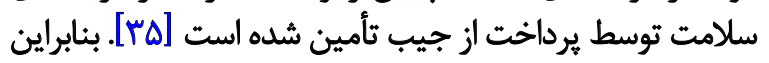

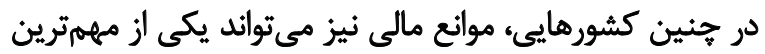

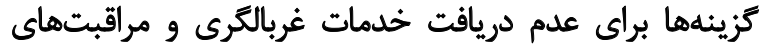

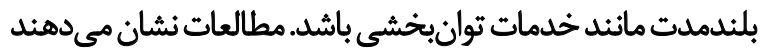

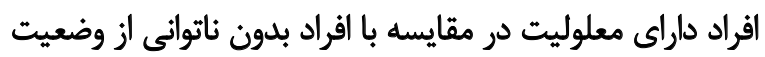

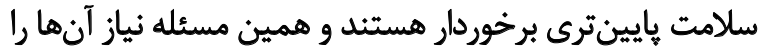

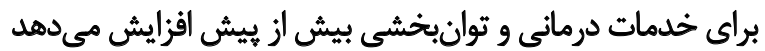

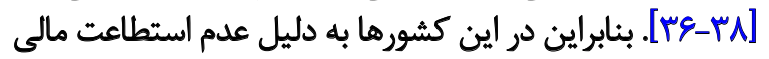

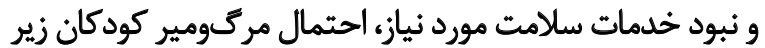

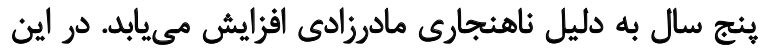

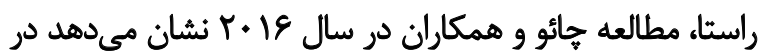

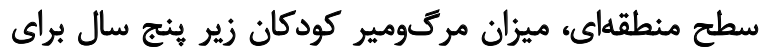

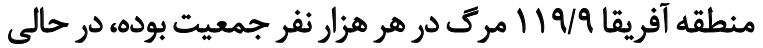

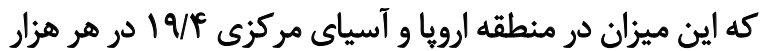

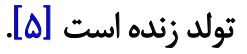

در كشورهاى با درآمد بايين كه دولت سهم كمترى از

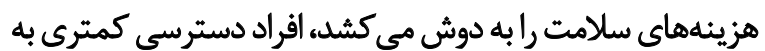




$$
\begin{aligned}
& \text { علـى كاظمسى كاريانسى و مسـلم صوفـى؛ مديريست يــروزه: } \\
& \text { بهـزاد كرمىمتيــن و شـاهين ســلطانى. }
\end{aligned}
$$

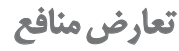

بنابر اظهار نويسندكان، اين مقاله تعارض منافع ندارد.

\section{نتيجهَيرى}

نتايج اين مطالعه نشان داد ميانكين تعداد سالهاي تحصيل

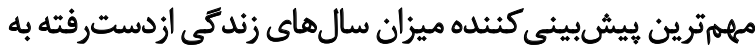

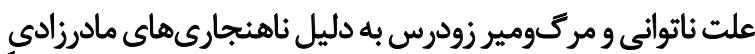

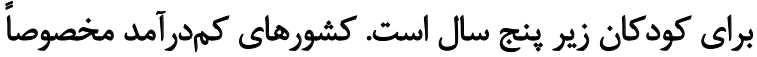

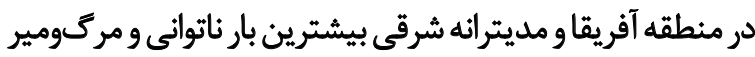

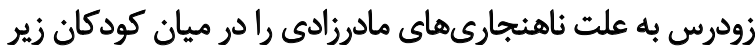
ينج سال متحمل مىشوند.

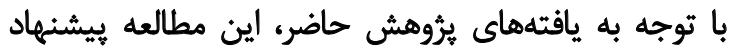

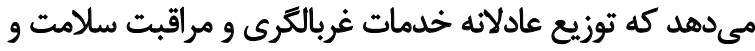

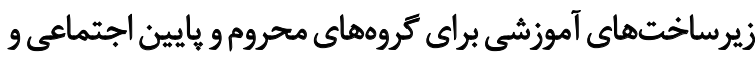

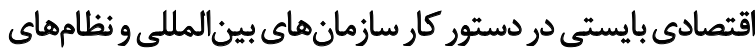

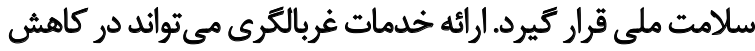

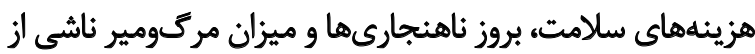
آنها در كودكان زير ينج سال تأثير كذار باشد.

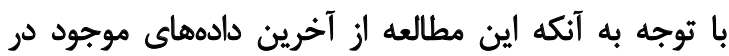

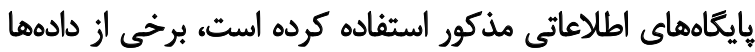

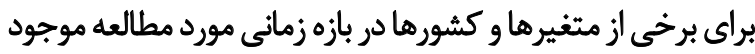

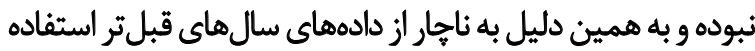

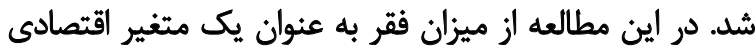

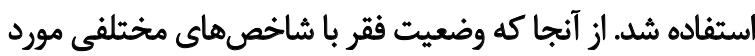

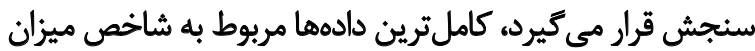
فقر بود و به همين دليل از اين شاخص استفاده شدرد

مالاحظات اخالاقى

بيروى از أصول اخلاق يثوبهش

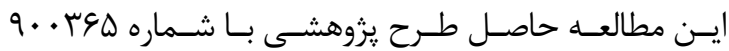

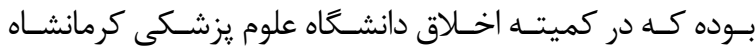

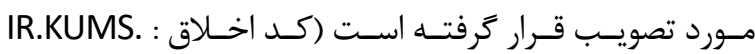

.(REC.1399.182

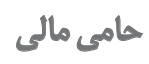

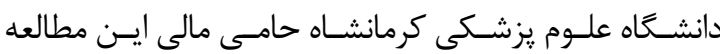

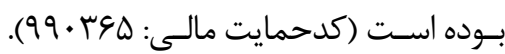

$$
\text { مشاركت نويسندكًان }
$$

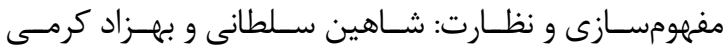

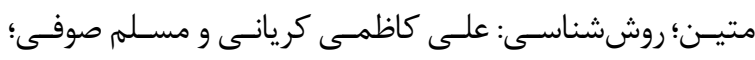

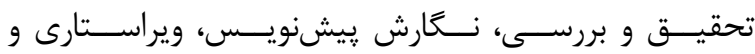

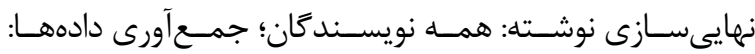

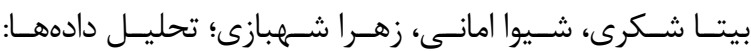




\section{References}

[1] DeSilva M, Munoz FM, Mcmillan M, Kawai AT, Marshall H, Macartney KK, et al. Congenital anomalies: Case definition and guidelines for data collection, analysis, and presentation of immunization safety data. Vaccine. 2016; 34(49):6015-26. [DOI:10.1016/j. vaccine.2016.03.047] [PMID] [PMCID]

[2] Hematyar M, Khajouie P. [Prevalence of congenital anomalies in 1000 live births in Javaheri Hospital, Tehran, 2004 (Persian)]. Medical Sciences. 2005; 15(2):75-8. http://tmuj.iautmu.ac.ir/ article-1-269-en.html

[3] World Health Organization. Congenital anomalies [Internet]. 2020 [Updated 2020 December 1]. Available from: https://www. who.int/news-room/fact-sheets/detail/congenital-anomalies

[4] Christianson A, Howson CP, Modell B. March of dimes: Global report on birth defects, the hidden toll of dying and disabled children [Internet]. 2006 [Updated 2006]. Available from: https:// www.marchofdimes.org/materials/global-report-on-birth-defects-the-hidden-toll-of--d2unzZI5_VWOaLZnw6iHcx7hbpMWtWzTuIOU3DabcVY.pdf

[5] Vatankhah S, Jalilvand M, Sarkhosh S, Azarmi M, Mohseni M. Prevalence of congenital anomalies in Iran: A review article. Iranian Journal of Public Health. 2017; 46(6):733-43. https://ijph. tums.ac.ir/index.php/ijph/article/view/10075

[6] Rahnama F, Hashemiyan M, Akbarzadeh R, Akabari A. [The incidence of apparent congenital anomalies in neonates in Mobini Maternity Hospital in Sabzevar Iran in 2005-6 (Persian)]. Journal of Sabzevar University of Medical Sciences. 2008; 15(4):231-6. http://jsums.medsab.ac.ir/article_121.html

[7] Daliri S, Sayehmiri K, Asadollahi Kh, Rezaei N, Saroukhani D, Karimi A. Prevalence of congenital anomalies in Iran: A systematic review and meta-analysis. Iranian Journal of Neonatology. 2018; 9(2):21-32. https://ijn.mums.ac.ir/article_10859.html

[8] Mashhadi Abdolahi H, Kargar Maher MH, Afsharnia F, Dastgiri S. Prevalence of congenital anomalies: A community-based study in the Northwest of Iran. International Scholarly Research Notices. 2014; 2014:920940. [DOI:10.1155/2014/920940] [PMID] [PMCID]

[9] Dastgiri S, Heidarzadeh M, Dastgiri A. Tabriz registry of congenital anomalies: A report of 10 years of monitoring birth defects in Iran. Congenital Anomalies. 2013; 53(2):98-9. [DOI:10.1111/ cga.12009] [PMID]

[10] Sedighi I, Nouri Sh, Sabzehei MK, Sangestani M, Mohammadi Y, Amiri J, et al. Determining the risk factors of congenital anomalies of newborns in Hamadan province. Journal of Comprehensive Pediatrics. 2020; 11(2):e90907. [DOI:10.5812/compreped.90907]

[11] Abdou MSM, Ali Reda Sherif A, Helmy Wahdan IM, Saad El din Ashour Kh. Pattern and risk factors of congenital anomalies in a pediatric university hospital, Alexandria, Egypt. Journal of the Egyptian Public Health Association. 2019; 94(1):3. [DOI:10.1186/s42506-018-0004-3] [PMID] [PMCID]

[12] Taye M, Afework M, Fantaye W, Diro E, Worku A. Factors associated with congenital anomalies in Addis Ababa and the Amhara Region, Ethiopia: A case-control study. BMC Pediatrics. 2018; 18(1):142. [DOI:10.1186/s12887-018-1096-9] [PMID] [PMCID]
[13] Almeida LFG, Araujo Júnior E, Crott GC, Okido MM, Berezowsk AT, Duarte G, et al. Epidemiological Risk Factors and perinatal out comes of congenital anomalies. Revista Brasileira de Ginecologia e Obstetrícia. 2016; 38(07):348-55. [DOI:10.1055/s-0036-1586160] [PMID]

[14] Ameen SK, Kareem Alalaf Sh, Shabila NP. Pattern of congenital anomalies at birth and their correlations with maternal characteristics in the maternity teaching hospital, Erbil city, Iraq. BMC Pregnancy and Childbirth. 2018; 18(1):501. [DOI:10.1186/s12884-018-2141-2] [PMID] [PMCID]

[15] Kurdi AM, Majeed-Saidan MA, Al Rakaf MS, AlHashem AM, Botto LD, Baaqeel HS, et al. Congenital anomalies and associated risk factors in a Saudi population: A cohort study from pregnancy to age 2 years. BMJ Open. 2019; 9(9):e026351. [DOI:10.1136/bmjopen-2018-026351] [PMID] [PMCID]

[16] GBD 2016 DALYs and HALE Collaborators. Global, regional, and national Disability-Adjusted Life-Years (DALYs) for 333 diseases and injuries and healthy life expectancy (HALE) for 195 countries and territories, 1990-2016: A systematic analysis for the Global Burden of Disease Study 2016. The Lancet. 2017; 390(10100):1260-344. [DOI:10.1016/S0140-6736(17)32130-X]

[17] GBD 2015 Child Mortality Collaborators. Global, regional, national, and selected subnational levels of stillbirths, neonatal, infant, and under-5 mortality, 1980-2015: A systematic analysis for the Global Burden of Disease Study 2015. The Lancet. 2016; 388(10053):1725-74. [DOI:10.1016/S0140-6736(16)31575-6] [PMID] [PMCID]

[18] Clark JD, Mossey PA, Sharp L, Little J. Socioeconomic status and orofacial clefts in Scotland, 1989 to 1998. The Cleft PalateCraniofacial Journal. 2003; 40(5):481-5. [DOI:10.1597/15451569_2003_040_0481_ssaoci_2.0.co_2] [PMID]

[19] Mortensen LH, Helweg-Larsen K, Nybo Andersen AM. Socioeconomic differences in perinatal health and disease. Scandinavian Journal of Public Health. 2011; 39 (7 Suppl):110-4. [DOI:10.1177/1403494811405096] [PMID]

[20] Soltani Sh, Takian AH, Akbari Sari A, Kamali M, Majdzadeh R, Karami Matin B. [Disregarded health problems of people with disabilities: A qualitative study of policymakers' perspective (Persian)]. Archives of Rehabilitation. 2019; 20(2):136-49. [DOI:10.32598/ri.20.2.136]

[21] Mofizul Islam M. Social determinants of health and related inequalities: Confusion and implications. Frontiers in Public Health. 2019, 7:11. [DOI:10.3389/fpubh.2019.00011] [PMID] [PMCID]

[22] Andermann A, Collaboration C. Taking action on the social determinants of health in clinical practice: A framework for health professionals. CMAJ. 2016; 188(17-18):E474-83. [DOI:10.1503/cmaj.160177] [PMID] [PMCID]

[23] GBD 2015 DALYs and HALE Collaborators. Global, regional, and national Disability-Adjusted Life-Years (DALYs) for 315 diseases and injuries and healthy life expectancy (HALE), 1990-2015: A systematic analysis for the Global Burden of Disease Study 2015. The Lancet. 2016; 388(10053):1603-58. [DOI:10.1016/S0140-6736(16)31460-X] [PMID] [PMCID]

[24] 24. Murray CJ, Barber RM, Foreman KJ, Ozgoren AA, Abd-Allah F, Abera SF, et al. Global, regional, and national disability-adjusted life years (DALYs) for 306 diseases and injuries and healthy life expectancy (HALE) for 188 countries, 1990-2013: quantifying the epidemiological transition. 2015; 386(10009):2145-91. 
[25] Hutto N, Waldfogel J, Kaushal N, Garfinkel I. Improving the measurement of poverty. Social Service Review. 2011; 85(1):39-74. [DOI:10.1086/659129] [PMID] [PMCID]

[26] Malhotra C, Do YK. Public health expenditure and health system responsiveness for low-income individuals: Results from 63 countries. Health Policy and Planning. 2017; 32(3):314-9. [DOI:10.1093/heapol/czw127] [PMID]

[27] Institute for Health Metrics and Evaluation. Global both sexes, all ages, 2019, DALYs [Internet]. 2019 [Updated 2019]. Available from: https://vizhub.healthdata.org/gbd-compare/

[28] UNESCO Institute of Statistics. Literacy [Internet]. 2019 [Updated 2019]. Available from: http://uis.unesco.org/en/topic/ literacy

[29] Soltani Sh, Takian AH, Akbari Sari A, Majdzadeh R, Kamali M. Cultural barriers in access to healthcare services for people with disability in Iran: A qualitative study. Medical Journal of the Islamic Republic of Iran. 2017; 31:51. [DOI:10.14196/mjiri.31.51] [PMID] [PMCID]

[30] Pawluk MS, Campaña H, Gili JA, Comas B, Giménez LG, Villalba MI, et al. [Adverse social determinants and risk for congenital anomalies (English-Spanish)]. Archivos Argentinos de Pediatría. 2014; 112(3):215-23. [DOI:10.5546/aap.2014.eng.215] [PMCID]

[31] Vrijheid M, Dolk H, Stone D, Abramsky L, Alberman E, Scott JES. Socioeconomic inequalities in risk of congenital anomaly. Archives of Disease in Childhood. 2000; 82(5):349-52. [DOI:10.1136/adc.82.5.349] [PMID] [PMCID]

[32] Sitkin NA, Ozgediz D, Donkor P, Farmer DL. Congenital anomalies in low- and middle-income countries: The unborn child of global surgery. World Journal of Surgery. 2015; 39(1):36-40. [DOI:10.1007/s00268-014-2714-9] [PMID] [PMCID]

[33] Liu L, Oza Sh, Hogan D, Chu Y, Perin J, Zhu J, et al. Global, regional, and national causes of under-5 mortality in 2000-15: An updated systematic analysis with implications for the Sustainable Development Goals. The Lancet. 2016; 388(10063):3027-35. [DOI:10.1016/S0140-6736(16)31593-8] [PMID] [PMCID]

[34] Olusanya BO, Wright SM, Nair MKC, Boo NY, Halpern R, Kuper $\mathrm{H}$, et al. Global burden of childhood epilepsy, intellectual disability, and sensory impairments. Pediatrics. 2020; 146(1):e20192623. [DOI:10.1542/peds.2019-2623] [PMID]

[35] Evans DB, Etienne C. Health systems financing and the path to universal coverage. Bulletin of the World Health Organization. 2010; 88(6):402. [DOI:10.2471/BLT.10.078741] [PMID] [PMCID]

[36] Morgan JP, Minihan PM, Stark PC, Finkelman MD, Yantsides KE, Park A, et al. The oral health status of 4,732 adults with intellectual and developmental disabilities. Journal of the American Dental Association. 2012; 143(8):838-46. [DOI:10.14219/jada. archive.2012.0288] [PMID]

[37] Gregg EW, Beckles GL, Williamson DF, Leveille SG, Langlois JA, Engelgau MM, et al. Diabetes and physical disability among older U.S. adults. Diabetes Care. 2000; 23(9):1272-7. [DOI:10.2337/diacare.23.9.1272] [PMID]

[38] Maart S, Jelsma J. Disability and access to health care - a community based descriptive study. Disability and Rehabilitation. 2014; 36(18):1489-93. [DOI:10.3109/09638288.2013.807883] [PMID]
[39] Sanogo NA, Fantaye AW, Yaya S. Universal health coverage and facilitation of equitable access to care in Africa. Frontiers in Public Health. 2019; 7:102. [DOI:10.3389/fpubh.2019.00102] [PMID] [PMCID] 
This Page Intentionally Left Blank 